\title{
Polycyanurates via Molecular Dynamics: In-Situ Crosslinking from Di(Cyanate Ester) Resins and Model Validation through Comparison to Experiment
}

Levi M. J. Moore, ${ }^{1 *}$ Neil D. Redeker, ${ }^{2}$ Andrea R Browning, ${ }^{3}$ Jeffrey M. Sanders, ${ }^{4}$ Kamran B. Ghiassi $^{1}$

1 Air Force Research Laboratory, Aerospace Systems Directorate, Edwards AFB, California 93524, United States

2 Exquadrum, Incorporated, Air Force Research Laboratory, Edwards AFB, California 93524, United States

${ }^{3}$ Schrödinger LLC, Portland, Oregon 97204, United States

${ }^{4}$ Schrödinger LLC, New York City, New York 10036, United States

* Corresponding Author: levi.moore.1@us.af.mil

Contents

1. SMARTS patterns used for crosslinking and RDF analysis

2. RDF Data 


\section{SMARTS Patterns Used for Crosslinking and RDF Analysis}

A crosslinking/bond formation step is defined in one reaction as one bond breaking and another bond forming. The SMARTS patterns used for the crosslinking steps are detailed below, the A and B SMARTS patterns being those input to the crosslinking algorithm to yield the reaction product of the next step.
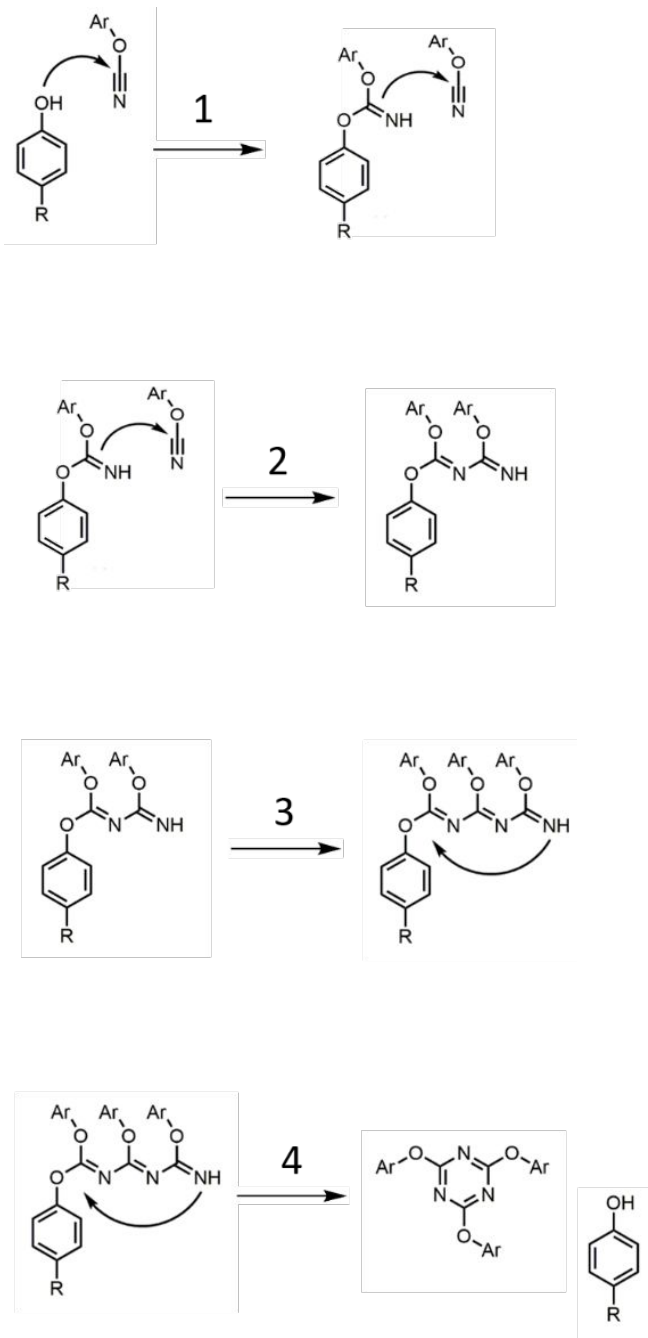

1:

$[\mathrm{N}] \#[\mathrm{C}]$ $[\mathrm{H}][\$([\mathrm{O}] \mathrm{c} 1 \mathrm{ccc}(\mathrm{CCCCCCCCC}) \mathrm{cc} 1)]$ Rate $=1$

2:

$[\mathrm{N}] \#[\mathrm{C}]$ $[\mathrm{H}][\$([\mathrm{~N}]=\mathrm{COc} 1 \mathrm{ccc}(\mathrm{CCCCCCCCC}) \mathrm{cc} 1)]$ Rate $=1$

3:

$[\mathrm{N}] \#[\mathrm{C}]$ $[\mathrm{H}][\$([\mathrm{~N}]=\mathrm{CN}=\mathrm{COc} 1 \mathrm{ccc}(\mathrm{CCCCCCCCC}) \mathrm{cc} 1)]$ Rate $=1$

4: $[\mathrm{H}][\$([\mathrm{~N}]=\mathrm{CN}=\mathrm{CN}=\mathrm{COc} 1 \mathrm{ccc}(\mathrm{CCCCCCCCC}) \mathrm{cc} 1)]$ $[\$([\mathrm{O}] \mathrm{c} 1 \mathrm{ccc}(\mathrm{CCCCCCCCC}) \mathrm{cc} 1)][\$([\mathrm{C}]=\mathrm{NC}=\mathrm{NC}=\mathrm{N})]$ Rate $=100$

Figure S1. SMARTS patterns for Mechanism 1. Ar represents the aryl groups of the cyanate ester monomers. 

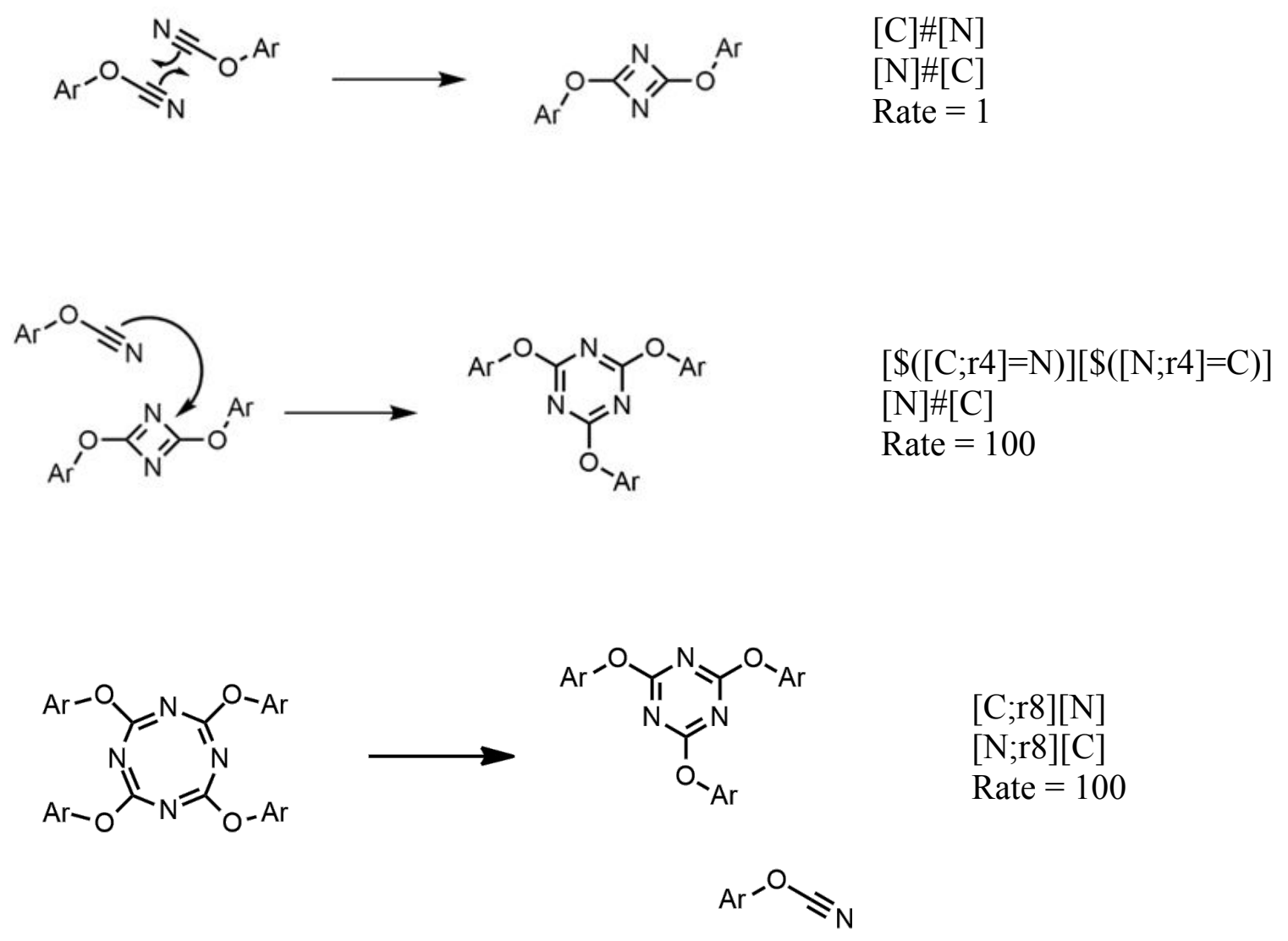

Figure S2. SMARTS patterns for Mechanism 2. Ar represents the aryl groups of the cyanate ester monomers. 
Table S1. ASLs used in RDF analyses.

\begin{tabular}{|c|c|}
\hline Moiety & SMARTS Pattern \\
\hline Water & water \\
\hline Phenol O & ((atom.ele O)) AND (“SMARTS. Oc1ccc(CCCCCCCCC)cc1”) \\
\hline Cyanate $\mathrm{N}$ & “SMARTS. [NX1]” \\
\hline Triazine $\mathrm{N}$ & "SMARTS. n" \\
\hline Ether & ((atom.ele O)) AND (“SMARTS.cOc”) \\
\hline Bridgehead & $\begin{array}{l}\text { BADCy: "SMARTS. [CX4;H0]" } \\
\text { LECy: "SMARTS. [CX4;H1]”" } \\
\text { SiMCy: "SMARTS. [Si]" }\end{array}$ \\
\hline Cyclodimer $\mathrm{N}$ & "SMARTS. [N;r4]" \\
\hline Imine $\mathrm{N}$ & "SMARTS. [N;H1]" \\
\hline
\end{tabular}




\section{RDF Data}
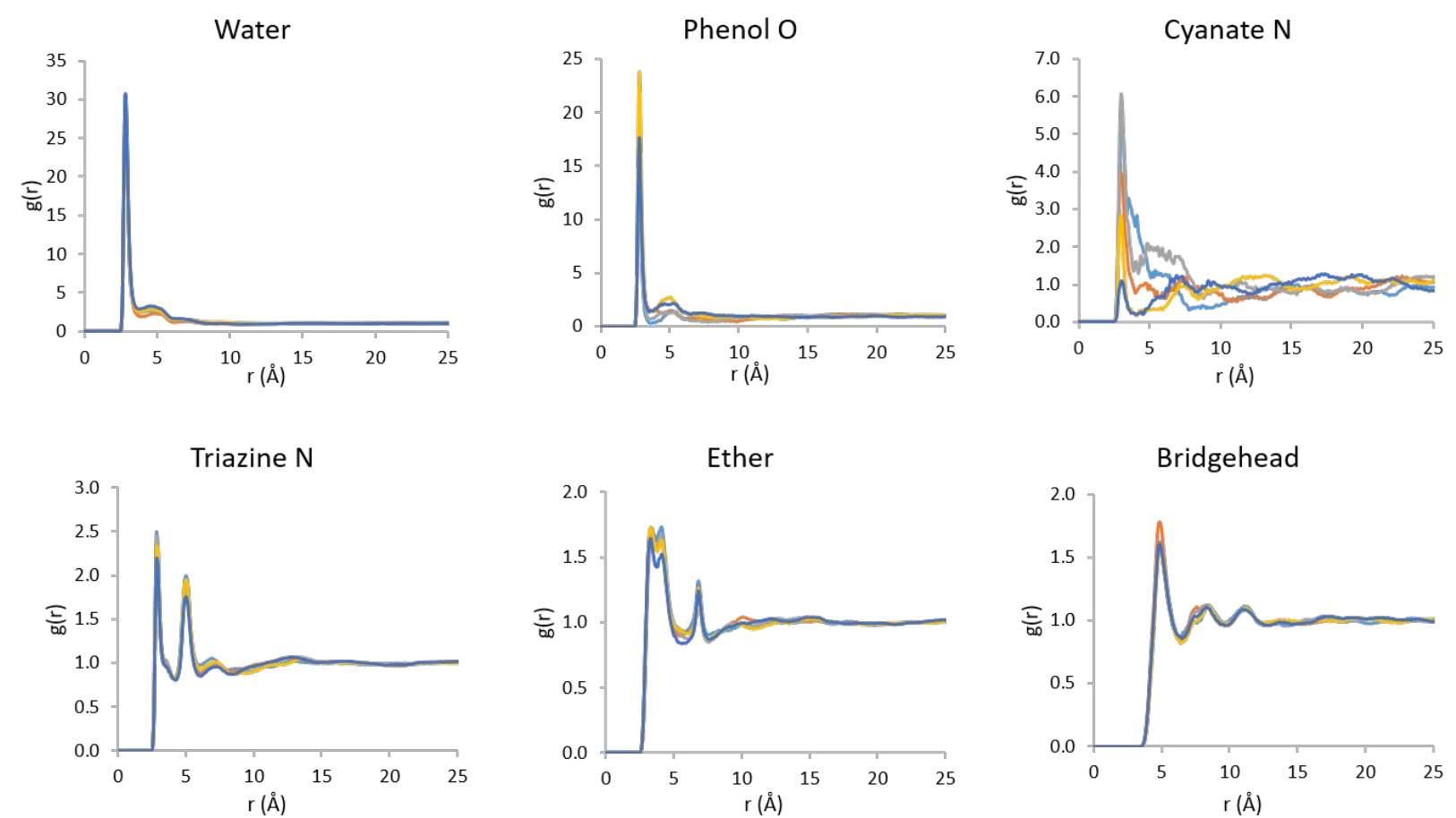

Figure S3. All RDF plots of crosslinked BADCy systems subjected to penetrant loading and subsequent relaxation. Each color represents a separate replicate.
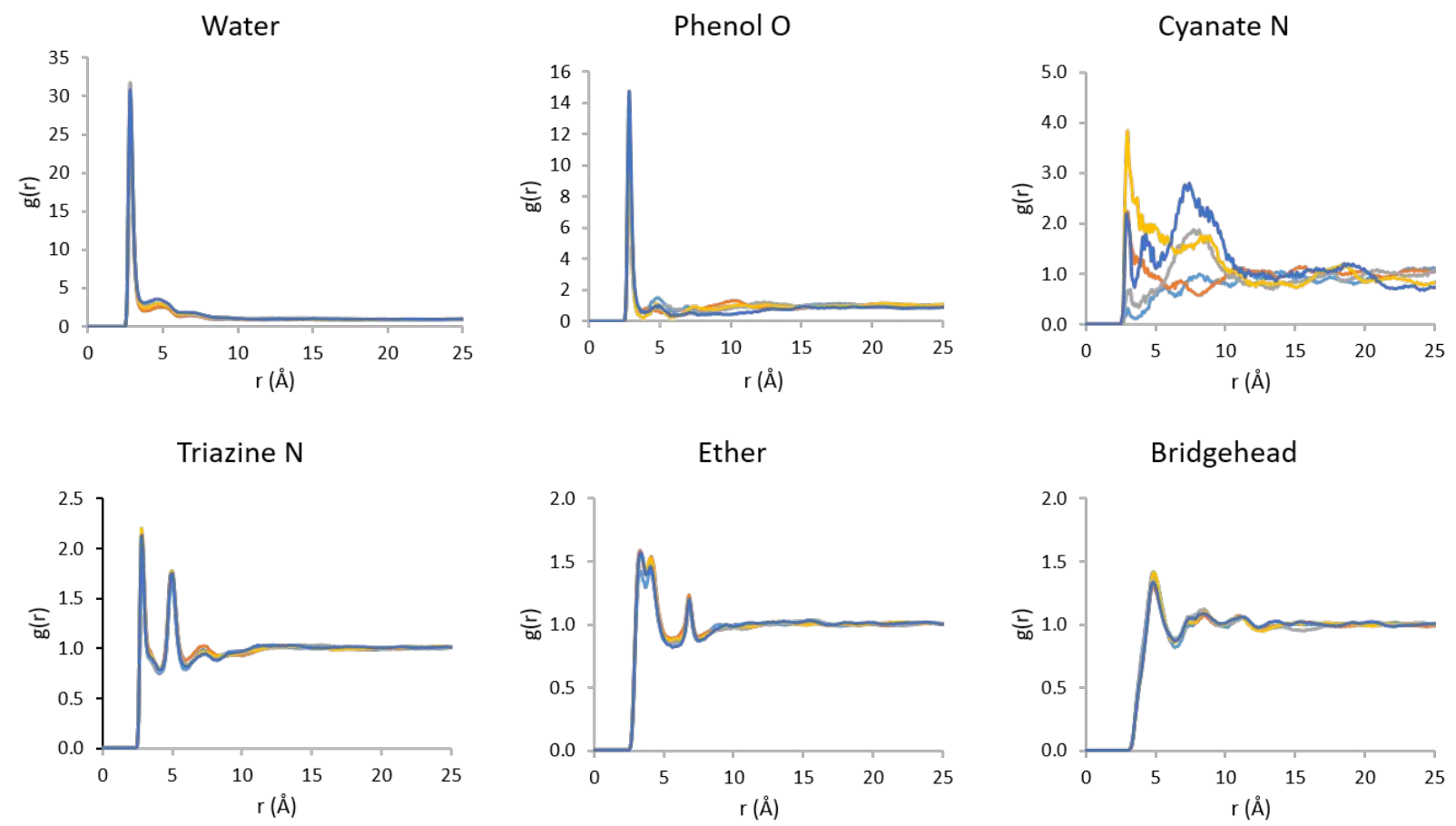

Figure S4. All RDF plots of crosslinked LECy systems subjected to penetrant loading and subsequent relaxation. Each color represents a separate replicate. 

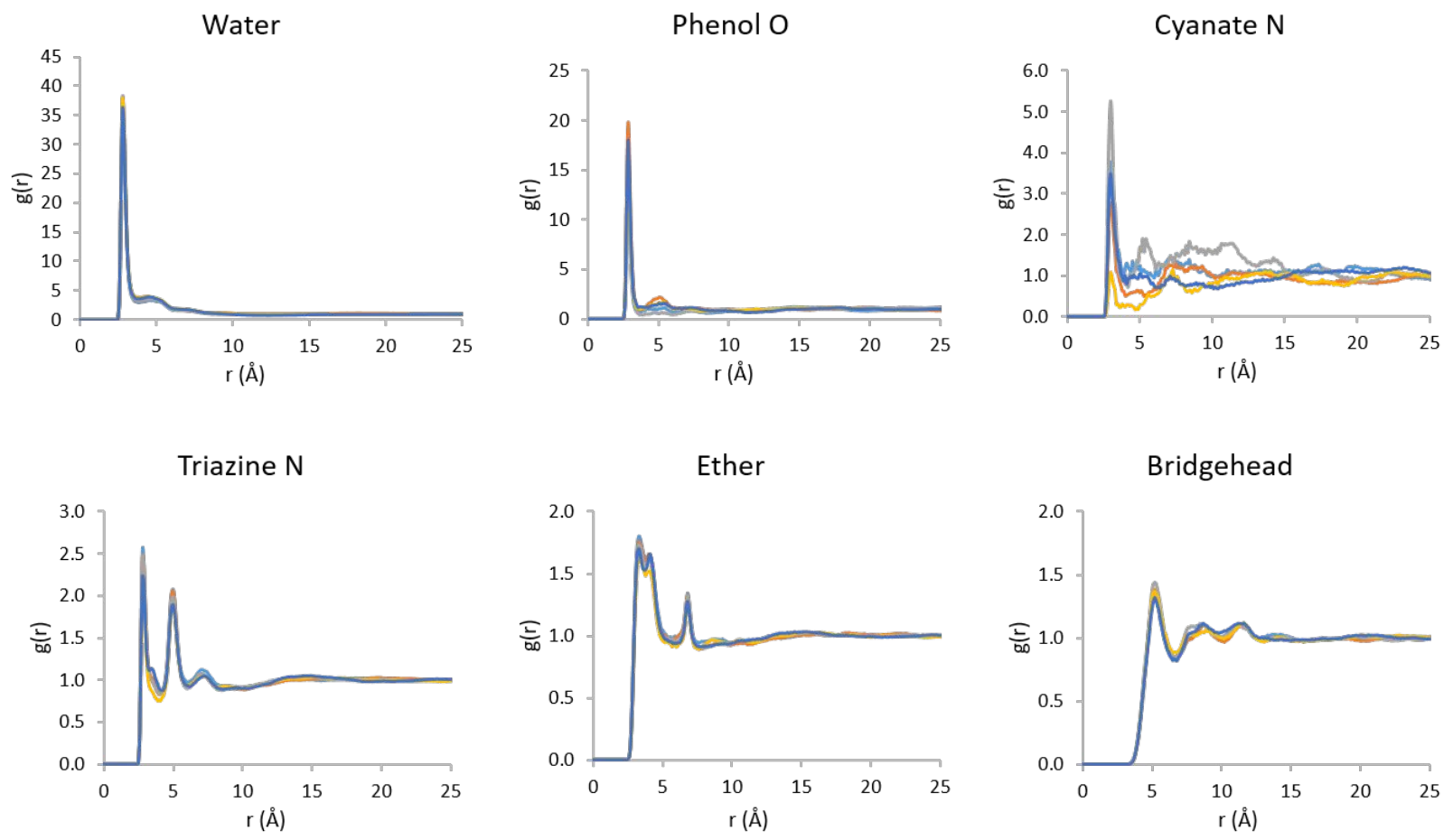

Figure S5. All RDF plots of crosslinked SiMCy systems subjected to penetrant loading and subsequent relaxation. Each color represents a separate replicate.
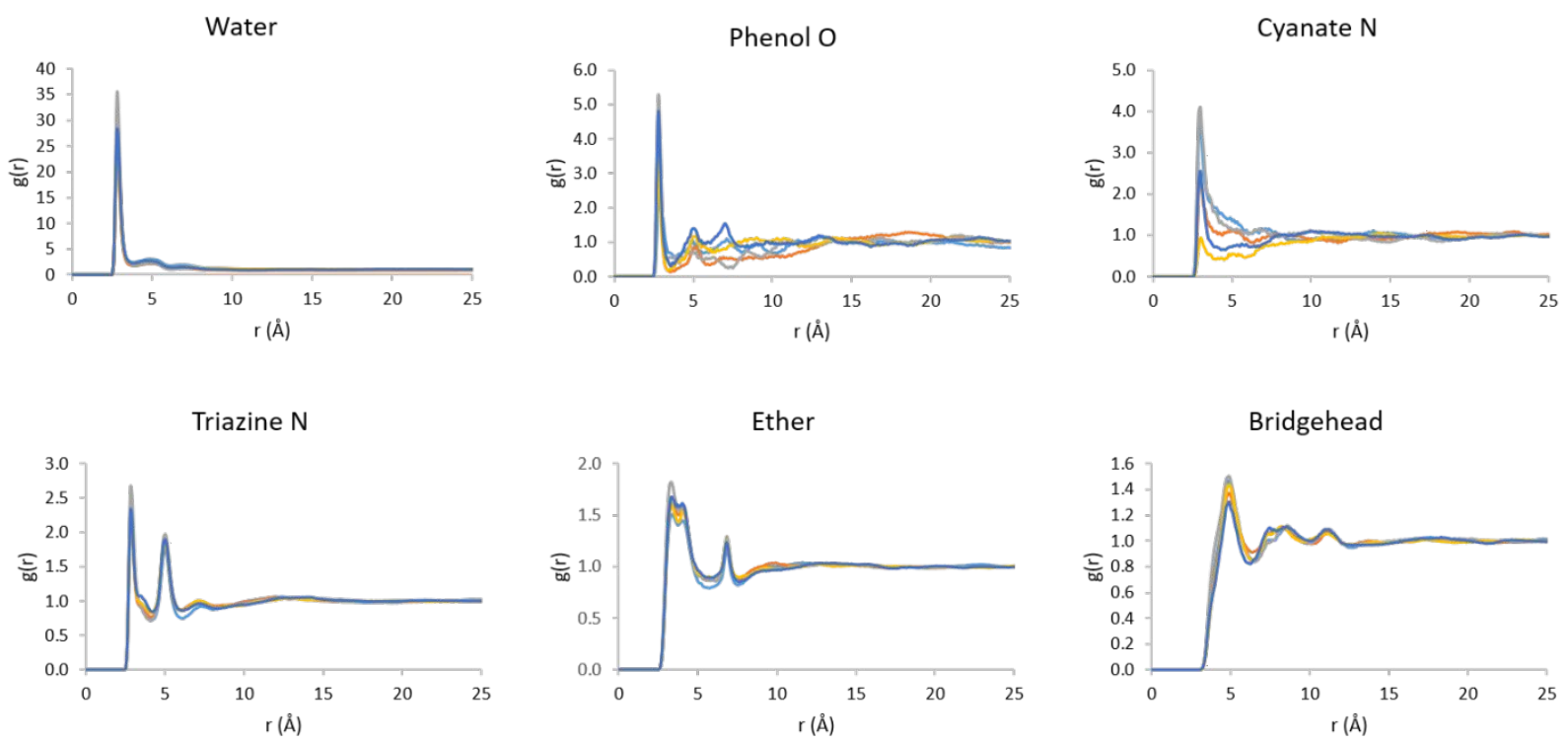

Figure S6. All RDF plots of LECy systems crosslinked via the chain mechanism (Mechanism 1). Each color represents a separate replicate. All are indistinguishable from Mechanism 2, aside from the "Phenol O" plot, due to fewer free phenol moieties in the system, an expected feature of the crosslinking mechanism. 
imine $\mathrm{N}$

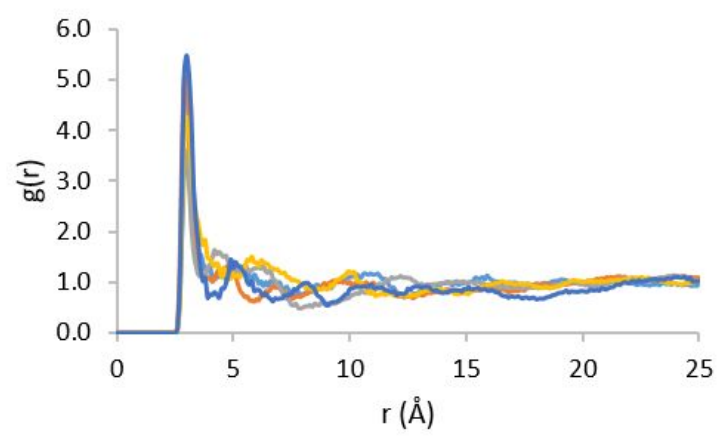

cyclodimer $\mathrm{N}$

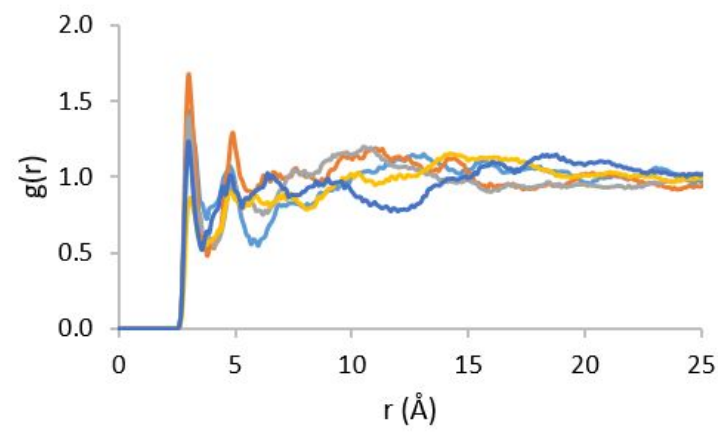

Figure S7. RDF plots of residual moieties from the crosslinking algorithm, the nitrogen in the cyclodimer from Mechanism 1 (left) and the imine nitrogen from Mechanism 2 (right).

Table S2. Comparison of coordination numbers of the selected moieties compared to water in the RDF of LECy systems crosslinked via the two different crosslinking mechanisms. All coordination numbers are not statistically different between the two systems, aside from the oxygen from the free phenol ("Phenol O"). This is due to a lower incidence of the free phenol in the system due to its incorporation into the polymer matrix by the crosslinking mechanism. The values from the imine $\mathrm{N}$ and phenolic $\mathrm{O}$ crosslinked via Mechanism 1 together approximately equal the value for the free phenol in the system crosslinked via Mechanism 2.

\begin{tabular}{|l|c|c|}
\hline Moiety & \multicolumn{2}{|c|}{ Coordination Number } \\
\hline Water & Mechanism 1 & Mechanism 2 \\
\hline Bridgehead & $1.93 \pm 0.24$ & $2.15 \pm 0.08$ \\
\hline Cyanate N & $1.43 \pm 0.32$ & $1.48 \pm 0.19$ \\
\hline Ether & $0.73 \pm 0.39$ & $0.51 \pm 0.57$ \\
\hline Phenol O & $1.18 \pm 0.27$ & $1.18 \pm 0.10$ \\
\hline Triazine N & $0.25 \pm 0.03$ & $0.80 \pm 0.08$ \\
\hline Cyclodimer N & $0.37 \pm 0.08$ & $0.37 \pm 0.04$ \\
\hline Imine N & -- & $0.20 \pm 0.06$ \\
\hline
\end{tabular}

